\title{
Urban-rural disparity of overweight/obesity distribution and its potential trend with breast cancer among Chinese women
}

\author{
Ying Gao ${ }^{1,2, *}$, Yubei Huang ${ }^{3, *}$, Fengju Song ${ }^{3}$, Hongji Dai ${ }^{3}$, Peishan Wang ${ }^{3}$, Haixin $\mathrm{Li}^{3}$, \\ Hong Zheng ${ }^{3}$, Henglei Dong ${ }^{3}$, Jiali Han ${ }^{4}$, Yaogang Wang ${ }^{1}$, Kexin Chen ${ }^{3}$ \\ ${ }^{1}$ Department of Health Service Management, School of Public Health, Tianjin Medical University, Tianjin, China \\ ${ }^{2}$ Health Management Center, Tianjin Medical University General Hospital, Tianjin, China \\ ${ }^{3}$ Department of Epidemiology and Biostatistics, Key Laboratory of Cancer Prevention and Therapy, Tianjin, Key Laboratory of \\ Breast Cancer Prevention and Therapy, Ministry of Education, National Clinical Research Center for Cancer, Tianjin Medical \\ University Cancer Institute and Hospital, Tianjin, China \\ ${ }^{4}$ Department of Epidemiology, Fairbanks School of Public Health, Simon Cancer Center, Indiana University, Indianapolis, \\ Indiana, USA \\ ${ }^{*}$ Co-first authors, these authors contributed equally to this work
}

Correspondence to: Kexin Chen, email: chenkexin@tijmu.edu.cn Yaogang Wang, email: wangyg@tmu.edu.cn

Keywords: overweight, obesity, disparity, breast cancer

Received: April 05, 2016

Accepted: July 19, 2016

Published: July 30, 2016

\section{ABSTRACT}

Objective: To evaluate the urban-rural disparity of overweight/obesity and explore its potential trend with breast cancer among Chinese women.

Results: The prevalence of overweight/obesity for Chinese rural women $(35.2 \%$, $\mathbf{2 9 . 2 \%}$ for overweight and $\mathbf{6 . 0 \%}$ for obesity) was significantly higher than that for Chinese urban women (33.4\%, $27.7 \%$ for overweight and $5.7 \%$ for obesity) $(P<\mathbf{0 . 0 0 1})$. For either rural or urban women, the prevalence of overweight/obesity was highest in north region, followed by east region for rural women and northeast region for urban women. For rural women, higher prevalence of overweight/ obesity was significantly positively associated with elder age, Han nationality, low level of education, no occupation, high family income, less number of family residents, insurance, and elder age at marriage. Similar positive associations were also found for urban women, except negative associations for high family income, less number of family residents, and elder age at marriage. A non-significant positive trend between overweight/obesity and breast cancer was found for rural women [odds ratio (OR): 1.06; 95\% confidence interval (CI): 0.87-1.29], but a significant positive trend for urban women (OR: 1.55; 95\% CI: 1.19-2.02).

Materials and Methods: A total of 1210762 participants were recruited from the Chinese National Breast Cancer Screening Program. Overweight and obesity were defined as body mass index (BMI) ranged $24.0-27.9 \mathrm{~kg} / \mathrm{m}^{2}$ and $B M I \geq 28.0 \mathrm{~kg} / \mathrm{m}^{2}$, respectively.

Conclusions: There was an obvious urban-rural disparity of overweight/obesity distribution among Chinese women, which could also lead to an obvious disparity of breast cancer distribution.

\section{INTRODUCTION}

Obesity is a known risk factor for various chronic diseases and mortality, including cancer, such as breast, colon and liver [1-3]. The Global Burden of Disease Study estimated that at least 2.1 billion adults were overweight, including 671 million people with obesity in 2013 [4]. In terms of global disability-adjusted life years (DALYs) in 2013, high body mass index (BMI) caused 4.4 million deaths and 134.0 million DALYs, and ranked as the third most important contributor to DALYs worldwide 5. Due to the increasing burden of disease caused by obesity [1-5], obesity has been defined as an independent chronic disease by the World Health Organization (WHO) [6]. As 
in the west, many Asian countries, for example China, are experiencing a steep rise in the prevalence of overweight/ obesity and obesity associated diseases in their populations [7-10].

Before 1990s, the etiologic studies of obesity mainly focused on dietary and behavioral factors [11, 12], few of them paid attention to the socio-demographic factors. Since Sobal and his colleague first systematically reviewed the relationship between socio-demographic factors and obesity [13], this topic had quickly become a hot spot in the field of obesity research. And several studies had found an inverse relationship between obesity and lower socioeconomic status, including low education, low income, no occupation, and so on [14-16]. In addition, the relationship had shown a potential region disparity, which indicated that different risk factors may be responsible for the different prevalence of obesity for different regions. However, most studies were conducted in developed countries [17-20], few of them were conducted in developing countries [21-23].

China, as the world's most populous country and the largest developing country, is also experiencing rapid economic, social, and cultural development, especially in urban areas. These rapid developments not only result in dramatic changes in lifestyles and dietary pattern, but also a greatly increased burden of obesity and other chronic diseases associated with these rapid changes [24, 25]. As the urban-rural disparity in socioeconomic level is enlarging, the urban-rural disparities in overweight/obesity and obesity-associated diseases are also increasing rapidly [26]. However, few studies focus on these urban-rural disparities in overweight/obesity and obesity-associated diseases, and explore the potential risk factors of these disparities, especially in the largest developing country.

Therefore, in the present study, we aim to examine the urban-rural disparity of overweight/obesity distribution and its potential trend with breast cancer among Chinese women, based on a large population-based survey of women from the Chinese National Breast Cancer Screening Program.

\section{RESULTS}

\section{Demographic characteristics of study participants}

A total of 1210762 participants were included in the final analyses. Among these participants, 397419 were urban residents and 813343 were rural residents. Among Chinese urban women, the proportions of Han nationality (96.6\%), a high level of education $(20.5 \%)$, having an occupation (94.8\%), high family income (26.7\%), less number of family residents $(67.7 \%)$, having insurance (80.4\%), and marriage at an older age (27.6\%) were significantly higher than those among Chinese rural women (the corresponding proportions were $87.1 \%, 2.7 \%$, $20.9 \%, 13.7 \%, 37.4 \%, 96.9 \%$, and $7.9 \%$, respectively).

\section{Prevalence of overweight/obesity}

According to Chinese criteria, the prevalence of overweight/obesity for Chinese rural women (35.2\%, $29.2 \%$ for overweight and $6.0 \%$ for obesity) was significantly higher than that for Chinese urban women (33.4\%, 27.7\% for overweight and 5.7\% for obesity) $(P<0.001$; Table 1$)$. According to WHO criteria, the corresponding prevalence of overweight/obesity for rural women $(23.1 \%)$ was still higher than that for urban women $(21.9 \%)(P<0.001$; Table 1$)$.

Due to the different sampling frame, we cannot directly compare the prevalence in this study with other Chinese nationally representative data published previously. However, a crude increasing trend for the prevalence of overweight/obesity could be observed across the data. Compared with the prevalence of overweight/ obesity from Chinese National Nutrition Survey 1992 (CNNS 1992), the prevalence of overweight/obesity crudely increased $5.6 \%$ for urban women (from $27.8 \%$ in 1992 to $33.4 \%$ in 2008 ) and $20.9 \%$ for rural women (from $14.3 \%$ in 1992 to $35.2 \%$ in 2008), respectively (Figure 1). In both urban and rural women in 2008, the ratios of overweight and obesity were approximately to 5.0 , which indicated a potentially huge obesity population would emerge from the overweight population either in Chinese urban or rural regions unless an urgent strategy is undertaken.

For either rural or urban women, the prevalence of overweight/obesity was highest in the northern region $(52.3 \%$ vs. $51.2 \%)$, followed by the eastern region for rural women $(39.7 \%)$ and north-eastern regions for urban women $(37.2 \%)$, respectively (Figure 2). According to the provincial distribution of overweight/obesity shown in Figure 3, the urban areas in Hebei, Tianjin, Beijing, Shanxi, Henan, and Liaoning showed obviously higher prevalence of overweight/obesity than urban areas in other provinces (Figure 3A). And the rural areas in Beijing, Shandong, Shanxi, Tianjin, Hebei, Henan, Jiangsu, and Xinjiang showed obviously higher prevalence of overweight/obesity than rural areas in other provinces (Figure 3B).

\section{Association between overweight/obesity and demographic characteristics}

As shown in Table 2 and Table 3, the prevalence of overweight/obesity showed a significantly increasing trend with age both for urban and rural women (both $P_{\text {trend }}$ $<0.001$ ). In rural areas, higher proportions of overweight/ obesity were more likely to be positively associated with Han nationality [vs. others nationality, odds ratio (OR): 1.18; 95\% confidence interval (CI): 1.16-1.20], high school education (vs. college or above, OR: $1.23 ; 95 \%$ CI: 1.20-1.27) and primary school education or below (vs. college or above, OR: 1.25; 95\% CI: 1.22-1.29), no 
Table 1: Prevalence of overweight/obesity among Chinese women in urban and rural areas according to Chinese criteria and WHO criteria

\begin{tabular}{|c|c|c|c|c|c|c|c|}
\hline \multirow[b]{2}{*}{ BMI } & \multicolumn{3}{|c|}{ Urban } & \multicolumn{3}{|c|}{ Rural } & \multirow[b]{2}{*}{$P$ value } \\
\hline & $N$ & $\begin{array}{c}\text { Crude } \\
\text { proportion, \% } \\
(95 \% \mathrm{CI})\end{array}$ & $\begin{array}{c}\text { Age- } \\
\text { standardized } \\
\text { proportion } \\
(\%)^{\mathrm{a}}\end{array}$ & $N$ & $\begin{array}{c}\text { Crude } \\
\text { proportion, \% } \\
(95 \% \mathrm{CI})\end{array}$ & $\begin{array}{c}\text { Age- } \\
\text { standardized } \\
\text { proportion } \\
(\%)^{\mathrm{a}}\end{array}$ & \\
\hline \multicolumn{8}{|l|}{ Chinese criteria } \\
\hline Underweight (BMI <18.5) & 18390 & $4.6(4.6-4.7)$ & 4.6 & 30648 & $3.8(3.7-3.8)$ & 3.8 & \\
\hline Normal $(\mathrm{BMI} \geq 18.5 \&<24.0)$ & 246299 & $62.0(61.8-62.1)$ & 61.0 & 496570 & $61.1(60.9-61.2)$ & 61.2 & \multirow{2}{*}{$<0.001$} \\
\hline Overweight $(\mathrm{BMI} \geq 24.0 \&<28.0)$ & 110083 & $27.7(27.6-27.8)$ & 28.4 & 237160 & $29.2(29.0-29.3)$ & 29.0 & \\
\hline Obesity (BMI $\geq 28.0$ ) & 22647 & $5.7(5.6-5.8)$ & 6.0 & 48965 & $6.0(6.0-6.1)$ & 6.0 & \\
\hline Overweight/Obesity (BMI $\geq 24.0)$ & 132730 & $33.4(33.3-33.5)$ & 34.4 & 286125 & $35.2(35.1-35.3)$ & 35.0 & $<0.001$ \\
\hline \multicolumn{8}{|l|}{ WHO criteria } \\
\hline Underweight (BMI <18.5) & 18390 & $4.6(4.6-4.7)$ & 4.6 & 30648 & $3.8(3.7-3.8)$ & 3.8 & \multirow{4}{*}{$<0.001$} \\
\hline Normal $(\mathrm{BMI} \geq 18.5 \&<25.0)$ & 292005 & $73.5(73.3-73.6)$ & 72.7 & 594630 & $73.1(73.0-73.2)$ & 73.2 & \\
\hline Overweight $(\mathrm{BMI} \geq 25.0 \&<30.0)$ & 78946 & $19.9(19.7-20.0)$ & 20.6 & 170043 & $20.9(20.8-21.0)$ & 20.8 & \\
\hline Obesity (BMI $\geq 30.0$ ) & 8078 & $2.0(2.0-2.1)$ & 2.1 & 18022 & $2.2(2.2-2.2)$ & 2.2 & \\
\hline Overweight/Obesity (BMI $\geq 25.0)$ & 87024 & $21.9(21.8-22.0)$ & 22.7 & 188065 & $23.1(23.0-23.2)$ & 23.0 & $<0.001$ \\
\hline
\end{tabular}

${ }^{a}$ Age-standardized to the 2000 Chinese population.

$P$ value, chi-square test.

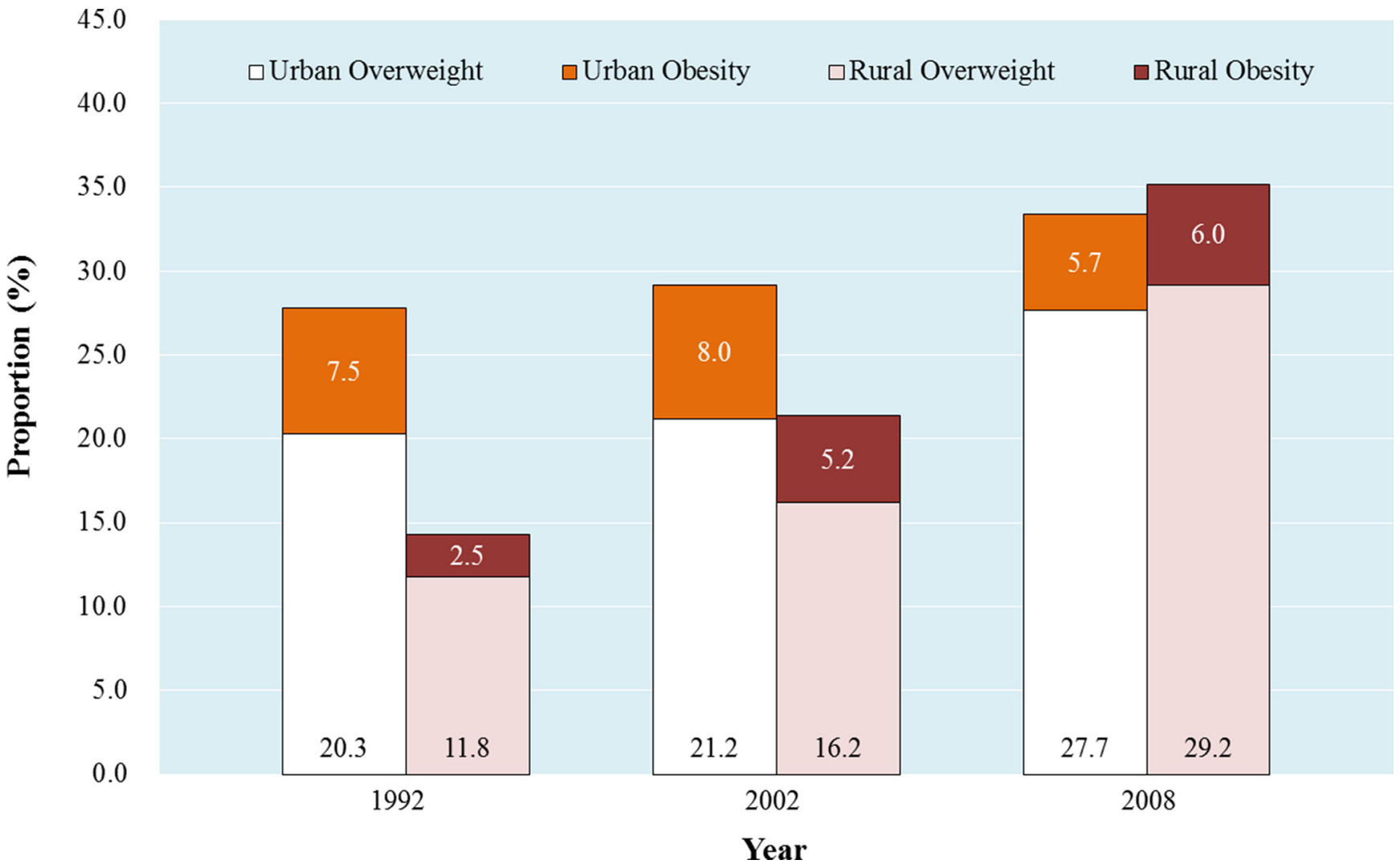

Figure 1: The women prevalence of overweight/obesity from Chinese National Nutrition Survey 1992 (CNNS 1992), Chinese National Nutritional and Health Survey 2002 (CNNHS 2002), and Chinese National Breast Cancer Screening Program 2008 (CNBCSP 2008). 
occupation (vs. with occupation, OR: 1.02; 95\% CI: $1.01-$ 1.03), family income of 1000-3000 RMB/month (vs. 1000 RMB/month or below, OR: 1.09; 95\% CI: 1.07-1.10) and $3000 \mathrm{RMB} / \mathrm{month}$ or above (vs. $1000 \mathrm{RMB} / \mathrm{month}$ or below, OR: 1.16; 95\% CI: 1.15-1.18), 1-3 family residents (vs. 4 family residents or above, OR: 1.04; 95\% CI: 1.03-1.05), with insurances (vs. no insurance, OR: 1.13 ; 95\% CI: $1.10-1.17)$, and the age at marriage being more than 26 years old (vs. 20 years or below, OR: 1.17; 95\% CI: $1.15-1.20$ ) and 21-25 years old (vs. 20 years or below, OR: 1.10; 95\% CI: 1.09-1.11).

Similar positive associations were also found among Chinese urban women, except negative association for family income of 1000-3000 RMB/month (vs. 1000 $\mathrm{RMB} /$ month or below, OR: 0.95 ; $95 \% \mathrm{CI}: 0.93-0.96$ ) and $3000 \mathrm{RMB} / \mathrm{month}$ or above (vs. $1000 \mathrm{RMB} / \mathrm{month}$ or below, OR: 0.83 ; 95\% CI: $0.81-0.85), 1-3$ family residents (vs. 4 family residents or above, OR: $0.71 ; 95 \%$ CI: $0.70-0.72)$, and the age at marriage being more than 26 years old (vs. 20 years or below, OR: $0.69 ; 95 \%$ CI: $0.67-0.70$ ) and $21-25$ years old (vs. 20 years or below, OR: 0.76 ; 95\% CI: 0.75-0.78).

\section{Trend between overweight/obesity and breast cancer}

A non-significant positive trend between overweight/ obesity and breast cancer was found for rural women (OR: 1.06; 95\% CI: 0.87-1.29), but a significant positive trend for urban women (OR: 1.55; 95\% CI: 1.19-2.02) (Table 4, Figure 3). After stratifying by socio-demographic variables, the positive trend was still observed between overweight/obesity and breast cancer in urban women, but not statistically significant in rural women (Supplementary Table S1).

\section{DISCUSSION}

In this study, we examined the urban-rural disparity of overweight/obesity distribution and its potential trend with breast cancer among Chinese women. Our findings suggested that the prevalence of overweight/obesity for Chinese rural women was significantly higher than that for Chinese urban women. This urban-rural disparity of overweight/obesity distribution could not only be found in the different regions, but also be found according to different demographic factors. Moreover, this urban-rural disparity of overweight/obesity distribution could also lead to a disparity of breast cancer distribution.

Several previous studies had also reported the disparity of overweight/obesity distribution. But most studies focused on the sex disparity or race/ethnic disparity in overweight/obesity distribution [14, 27], few studies focused on the urban-rural disparity. Several reasons could be responsible for this urban-rural disparity of overweight/ obesity distribution. The most important reason could be

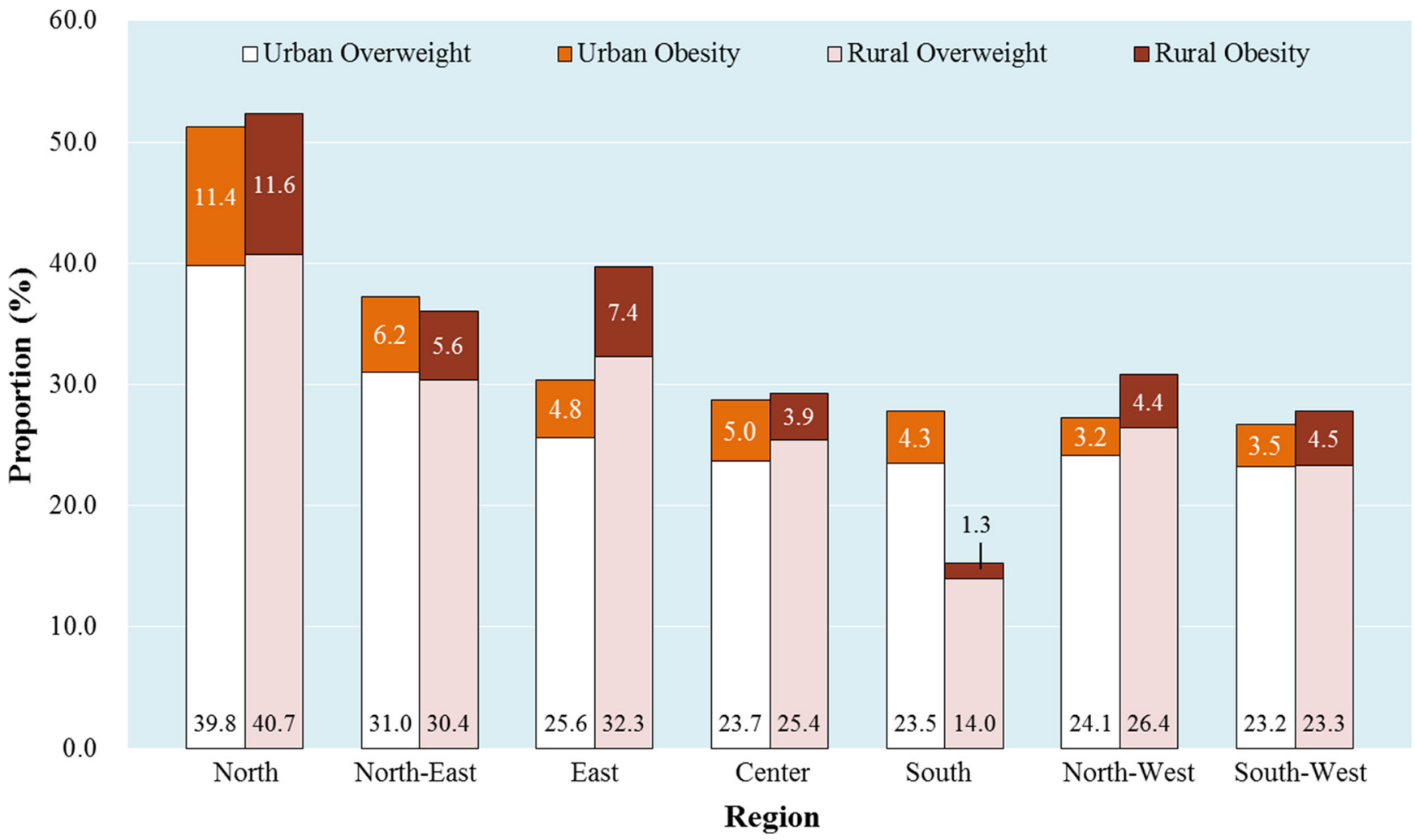

Figure 2: Regional prevalence of overweight/obesity among Chinese urban and rural women. Note: North included Beijing, Tianjin, Hebei, Inner Mongolia, Shanxi. North-East included Liaoning, Heilongjiang, Jilin. East included Shandong, Anhui, Jiangsu, Zhejiang, Shanghai, Fujian. Center included Jiangxi, Henan, Hubei, Hunan. South included Guangxi, Hainan. North-West included Gansu, Ningxia, Qinghai, Shaanxi, Xinjiang. South-West included Guizhou, Sichuan, Chongqing, Yunnan. 
Table 2: Prevalence of overweight/obesity among Chinese rural women according to different demographic characteristics

\begin{tabular}{|c|c|c|c|c|c|c|c|c|}
\hline \multirow[b]{2}{*}{ Variable } & \multicolumn{4}{|c|}{ BMI category $(N, \%)$} & \multirow[b]{2}{*}{$P_{1}$} & \multirow[b]{2}{*}{$P_{\text {trend }}$} & \multirow[b]{2}{*}{$P_{2}$} & \multirow[b]{2}{*}{ OR $(95 \% C I)^{a}$} \\
\hline & $\begin{array}{l}\text { Underweight } \\
\text { (BMI < 18.5) }\end{array}$ & $\begin{array}{c}\text { Normal } \\
(B M I \geq 18.5 \& \\
<24.0)\end{array}$ & $\begin{array}{c}\text { Overweight } \\
\text { (BMI } \geq \mathbf{2 4 . 0} \& \\
<\mathbf{2 8 . 0})\end{array}$ & $\begin{array}{c}\text { Obesity } \\
(\text { BMI } \geq 28.0)\end{array}$ & & & & \\
\hline \multicolumn{9}{|l|}{ Age (years) } \\
\hline $35-39$ & $10406(5.1)$ & $139496(68.1)$ & $46762(22.8)$ & $8076(3.9)$ & Ref. & & Ref. & 1.00 \\
\hline $40-44$ & $7033(3.5)$ & $125940(62.4)$ & $58121(28.8)$ & $10808(5.4)$ & $<0.001$ & & $<0.001$ & $1.42(1.40-1.44)$ \\
\hline $45-49$ & $5593(3.0)$ & $108517(58.4)$ & $59215(31.8)$ & $12607(6.8)$ & $<0.001$ & & $<0.001$ & $1.72(1.70-1.74)$ \\
\hline $50-54$ & $3511(3.2)$ & $61526(55.3)$ & 37378 (33.6) & $8830(7.9)$ & $<0.001$ & & $<0.001$ & $1.94(1.91-1.97)$ \\
\hline $55-59$ & $4105(3.7)$ & $61091(55.8)$ & $35684(32.6)$ & $8644(7.9)$ & $<0.001$ & $<0.001$ & $<0.001$ & $1.86(1.83-1.89)$ \\
\hline \multicolumn{9}{|l|}{ Nationality } \\
\hline Others & $4446(5.4)$ & $51925(62.8)$ & $21648(26.2)$ & $4640(5.6)$ & Ref. & & Ref. & 1.00 \\
\hline Han & $25696(3.6)$ & $431298(60.9)$ & $208436(29.4)$ & $42984(6.1)$ & $<0.001$ & - & $<0.001$ & $1.18(1.16-1.20)$ \\
\hline Unknown & $506(2.3)$ & 13347 (59.9) & $7076(31.8)$ & $1341(6.0)$ & & & & \\
\hline \multicolumn{9}{|l|}{ Education } \\
\hline$\geq$ College & $910(4.1)$ & $14321(65.3)$ & $5708(26.0)$ & $994(4.5)$ & Ref. & & Ref. & 1.00 \\
\hline High school & $14208(3.5)$ & $246659(61.3)$ & $117282(29.2)$ & $24021(6.0)$ & $<0.001$ & & $<0.001$ & $1.23(1.20-1.27)$ \\
\hline $\begin{array}{l}\leq \text { Primary } \\
\text { school }\end{array}$ & $14954(4.0)$ & $223367(60.5)$ & $108298(29.3)$ & $22879(6.2)$ & $<0.001$ & $<0.001$ & $<0.001$ & $1.25(1.22-1.29)$ \\
\hline Unknown & $576(2.9)$ & $12223(61.9)$ & $5872(29.7)$ & $1071(5.4)$ & & & & \\
\hline \multicolumn{9}{|l|}{ Occupation } \\
\hline Yes & $5840(3.4)$ & $104714(61.6)$ & $49256(29.0)$ & $10312(6.1)$ & Ref. & & Ref. & 1.00 \\
\hline No & $24543(3.9)$ & $387064(60.8)$ & $186370(29.3)$ & $38399(6.0)$ & $<0.001$ & - & 0.02 & $1.02(1.01-1.03)$ \\
\hline Unknown & $265(3.9)$ & $4792(70.0)$ & $1534(22.4)$ & $254(3.7)$ & & & & \\
\hline \multicolumn{9}{|c|}{ Family income (RMB/month) ${ }^{\mathrm{b}}$} \\
\hline$<1000$ & $10674(4.4)$ & $148122(61.6)$ & $67598(28.1)$ & $13971(5.8)$ & Ref. & & Ref. & 1.00 \\
\hline $1000-3000$ & $15253(3.4)$ & $271187(60.8)$ & $132595(29.7)$ & $27085(6.1)$ & $<0.001$ & & $<0.001$ & $1.09(1.07-1.10)$ \\
\hline$\geq 3000$ & $3432(3.1)$ & $66213(59.5)$ & $34192(30.7)$ & $7443(6.7)$ & $<0.001$ & $<0.001$ & $<0.001$ & $1.16(1.15-1.18)$ \\
\hline Unknown & $1289(8.3)$ & $11048(70.9)$ & $2775(17.8)$ & $466(3.0)$ & & & & \\
\hline \multicolumn{9}{|c|}{ Family resident (persons) } \\
\hline$\geq 4$ & $19144(3.8)$ & $304813(61.2)$ & $145239(29.2)$ & $28943(5.8)$ & Ref. & & Ref. & 1.00 \\
\hline $1-3$ & $11111(3.6)$ & $184361(60.5)$ & 89427 (29.4) & $19632(6.4)$ & $<0.001$ & - & $<0.001$ & $1.04(1.03-1.05)$ \\
\hline Unknown & $393(3.7)$ & $7396(69.3)$ & $2494(23.4)$ & $390(3.7)$ & & & & \\
\hline \multicolumn{9}{|l|}{ Insurance } \\
\hline No & $635(3.4)$ & $11905(64.0)$ & $5049(27.2)$ & $999(5.4)$ & Ref. & & Ref. & 1.00 \\
\hline Yes & $29801(3.8)$ & $480049(60.9)$ & $230573(29.3)$ & $47663(6.0)$ & $<0.001$ & $<0.001$ & $<0.001$ & $1.13(1.10-1.17)$ \\
\hline Unknown & $212(3.2)$ & $4616(69.2)$ & $1538(23.1)$ & $303(4.5)$ & & & & \\
\hline \multicolumn{9}{|l|}{ Marriage } \\
\hline Married & $29424(3.7)$ & $485010(61.0)$ & $232806(29.3)$ & $48002(6.0)$ & Ref. & & Ref. & 1.0 \\
\hline Others ${ }^{\mathrm{c}}$ & 477 (4.9) & $5752(59.0)$ & $2826(29.0)$ & $698(7.2)$ & $<0.001$ & - & 0.09 & $1.04(0.99-1.08)$ \\
\hline Unknown & 747 (8.9) & $5808(69.6)$ & $1528(18.3)$ & $265(3.2)$ & & & & \\
\hline \multicolumn{9}{|c|}{ Age at marriage (years) } \\
\hline$\leq 20$ & $8160(4.3)$ & $118448(62.2)$ & 53170 (27.9) & $10739(5.6)$ & Ref. & & Ref. & 1.00 \\
\hline $21-25$ & $19521(3.5)$ & $334697(60.8)$ & $163282(29.7)$ & $33098(6.0)$ & $<0.001$ & & $<0.001$ & $1.10(1.09-1.11)$ \\
\hline$\geq 26$ & $2544(4.0)$ & $37688(58.8)$ & 18964 (29.6) & $4851(7.6)$ & $<0.001$ & $<0.001$ & $<0.001$ & $1.17(1.15-1.20)$ \\
\hline Unknown & $423(5.2)$ & $5737(70.1)$ & $1744(21.3)$ & $277(3.4)$ & & & & \\
\hline
\end{tabular}

BMI, body mass index.

$P_{1}, P$ values of chi-square test for BMI distribution with four categories (Less weight, Normal, Overweight, Obesity).

$P_{2}, P$ values of univariate logistic regression for BMI distribution with two categories (Less weight/Normal, and Overweight/Obesity).

${ }^{\mathrm{a}} \mathrm{ORs}$ of Overweight/Obesity for the specific subgroups compared with the reference subgroup.

${ }^{\mathrm{b}}$ According to the exchange rate of Chinese RMB to U.S. dollar in 2008 ( $\left.1 \mathrm{RMB}=0.1611 \mathrm{USD}\right), 1000 \mathrm{RMB}=161.1 \mathrm{USD}, 3000 \mathrm{RMB}=$ 483.3 USD.

'Others included unmarried, separated, divorced and widowed. 
Table 3: Prevalence of overweight/obesity among Chinese urban women according to different demographic characteristics

\begin{tabular}{|c|c|c|c|c|c|c|c|c|}
\hline \multirow[b]{2}{*}{ Variable } & \multicolumn{4}{|c|}{ BMI category $(N, \%)$} & \multirow[b]{2}{*}{$P_{1}$} & \multirow[b]{2}{*}{$P_{\text {trend }}$} & \multirow[b]{2}{*}{$P_{2}$} & \multirow[b]{2}{*}{ OR $(95 \% C I)^{a}$} \\
\hline & $\begin{array}{l}\text { Underweight } \\
(\mathrm{BMI}<\mathbf{1 8 . 5})\end{array}$ & $\begin{array}{c}\text { Normal } \\
(\mathrm{BMI} \geq \mathbf{1 8 . 5} \\
\mathbf{\&}<\mathbf{2 4 . 0})\end{array}$ & $\begin{array}{c}\text { Overweight } \\
\text { (BMI } \geq \mathbf{2 4 . 0} \\
\boldsymbol{\&}<\mathbf{2 8 . 0})\end{array}$ & $\begin{array}{c}\text { Obesity } \\
(B M I \geq \\
28.0)\end{array}$ & & & & \\
\hline \multicolumn{9}{|l|}{ Age (years) } \\
\hline $35-39$ & $7023(7.3)$ & $69021(71.4)$ & $17608(18.2)$ & $2956(3.1)$ & Ref. & & Ref. & 1.00 \\
\hline $40-44$ & $3975(4.5)$ & $59002(66.8)$ & $21509(24.4)$ & $3802(4.3)$ & $<0.001$ & & $<0.001$ & $1.49(1.46-1.52)$ \\
\hline $45-49$ & $2609(3.5)$ & $44761(60.3)$ & $22374(30.1)$ & $4510(6.1)$ & $<0.001$ & & $<0.001$ & $2.10(2.05-2.14)$ \\
\hline $50-54$ & $1875(3.2)$ & $32354(55.8)$ & $19581(33.8)$ & $4204(7.2)$ & $<0.001$ & & $<0.001$ & $2.57(2.51-2.63)$ \\
\hline $55-59$ & $1358(3.2)$ & $21741(51.8)$ & $15218(36.3)$ & $3625(8.6)$ & $<0.001$ & & $<0.001$ & $3.02(2.94-3.09)$ \\
\hline $60-64$ & $908(3.8)$ & $12025(50.0)$ & $8835(36.8)$ & $2259(9.4)$ & $<0.001$ & & $<0.001$ & $3.17(3.08-3.27)$ \\
\hline $65-69$ & $642(4.5)$ & $7395(51.8)$ & $4958(34.7)$ & $1291(9.0)$ & $<0.001$ & $<0.001$ & $<0.001$ & $2.88(2.77-2.98)$ \\
\hline \multicolumn{9}{|l|}{ Nationality } \\
\hline Others & $570(4.8)$ & $7544(64.1)$ & $3076(26.1)$ & $575(4.9)$ & Ref. & & Ref. & 1.00 \\
\hline Han & $17755(4.6)$ & $237537(61.9)$ & $106521(27.8)$ & $21977(5.7)$ & $<0.001$ & - & $<0.001$ & $1.12(1.07-1.16)$ \\
\hline Unknown & $65(3.5)$ & $1218(65.3)$ & $486(26.1)$ & $95(5.1)$ & & & & \\
\hline \multicolumn{9}{|l|}{ Education } \\
\hline$\geq$ College & $4511(5.5)$ & $57946(71.3)$ & $16577(20.4)$ & $2292(2.8)$ & Ref. & & Ref. & 1.00 \\
\hline High school & $10522(4.5)$ & $144443(61.8)$ & $65568(28.0)$ & $13380(5.7)$ & $<0.001$ & & $<0.001$ & $1.69(1.66-1.72)$ \\
\hline$\leq$ Primary school & $3312(4.1)$ & $43391(53.3)$ & $27693(34.0)$ & $6937(8.5)$ & $<0.001$ & $<0.001$ & $<0.001$ & $2.45(2.40-2.51)$ \\
\hline Unknown & $45(5.3)$ & $519(61.3)$ & $245(28.9)$ & $38(4.5)$ & & & & \\
\hline \multicolumn{9}{|l|}{ Occupation } \\
\hline Yes & $17694(4.7)$ & $236749(62.9)$ & $102311(27.2)$ & $19862(5.3)$ & Ref. & & Ref. & 1.00 \\
\hline No & $659(3.3)$ & $9100(45.4)$ & $7544(37.6)$ & $2742(13.7)$ & $<0.001$ & - & $<0.001$ & $2.20(2.13-2.26)$ \\
\hline Unknown & $37(4.9)$ & $450(59.4)$ & $228(30.1)$ & $43(5.7)$ & & & & \\
\hline \multicolumn{9}{|c|}{ Family income (RMB/month) ${ }^{\mathrm{b}}$} \\
\hline$<1000$ & $3783(5.0)$ & $45669(59.9)$ & $21827(28.6)$ & $5013(6.6)$ & Ref. & & Ref. & 1.00 \\
\hline $1000-3000$ & $9592(4.5)$ & $130896(61.6)$ & $59830(28.1)$ & $12330(5.8)$ & $<0.001$ & & $<0.001$ & $0.95(0.93-0.96)$ \\
\hline$\geq 3000$ & $4916(4.6)$ & $68215(64.3)$ & $27739(26.2)$ & $5143(4.9)$ & $<0.001$ & $<0.001$ & $<0.001$ & $0.83(0.81-0.85)$ \\
\hline Unknown & $99(4.0)$ & $1519(61.6)$ & $687(27.9)$ & $161(6.5)$ & & & & \\
\hline \multicolumn{9}{|c|}{ Family resident (persons) } \\
\hline$\geq 4$ & $4810(4.5)$ & $59769(56.3)$ & $33532(31.6)$ & $7961(7.5)$ & Ref. & & Ref. & 1.00 \\
\hline $1-3$ & $12593(4.7)$ & $172258(64.0)$ & $70770(26.3)$ & $13481(5.0)$ & $<0.001$ & - & $<0.001$ & $0.71(0.70-0.72)$ \\
\hline Unknown & $987(4.4)$ & $14272(64.2)$ & $5781(26.0)$ & $1205(5.4)$ & & & & \\
\hline \multicolumn{9}{|l|}{ Insurance } \\
\hline No & $3281(5.9)$ & $34908(62.8)$ & $14514(26.1)$ & $2859(5.1)$ & Ref. & & Ref. & 1.00 \\
\hline Yes & $14123(4.4)$ & $197267(61.7)$ & $89756(28.1)$ & $18557(5.8)$ & $<0.001$ & - & $<0.001$ & $1.13(1.11-1.15)$ \\
\hline Unknown & $986(4.5)$ & $14124(63.8)$ & $5813(26.2)$ & $1231(5.6)$ & & & & \\
\hline \multicolumn{9}{|l|}{ Marriage } \\
\hline Married & $17408(4.6)$ & $236102(62.0)$ & $105663(27.7)$ & $21625(5.7)$ & Ref. & & Ref. & 1.00 \\
\hline Others $^{c}$ & $966(6.0)$ & $9920(61.2)$ & $4316(26.6)$ & $1009(6.2)$ & $<0.001$ & - & 0.13 & $0.97(0.94-1.01)$ \\
\hline Unknown & $16(3.9)$ & $277(67.6)$ & $104(25.4)$ & $13(3.2)$ & & & & \\
\hline \multicolumn{9}{|c|}{ Age at marriage (years) } \\
\hline$\leq 20$ & $1472(4.1)$ & $19944(56.1)$ & $11364(31.9)$ & $2789(7.8)$ & Ref. & & Ref. & 1.00 \\
\hline $21-25$ & $10373(4.3)$ & $151666(62.2)$ & 68025 (27.9) & $13765(5.6)$ & $<0.001$ & & $<0.001$ & $0.76(0.75-0.78)$ \\
\hline$\geq 26$ & $6059(5.5)$ & $69455(63.3)$ & $28616(26.1)$ & $5622(5.1)$ & $<0.001$ & $<0.001$ & $<0.001$ & $0.69(0.67-0.70)$ \\
\hline Unknown & $486(5.9)$ & $5234(63.3)$ & $2078(25.1)$ & $471(5.7)$ & & & & \\
\hline
\end{tabular}

BMI, body mass index.

$P_{1}, P$ values of chi-square test for BMI distribution with four categories (Less weight, Normal, Overweight, Obesity).

$P_{2}, P$ values of univariate logistic regression for BMI distribution with two categories (Less weight/Normal, and Overweight/ Obesity).

${ }^{a}$ ORs of Overweight/Obesity for the specific subgroups compared with the reference subgroup.

${ }^{\mathrm{b}}$ According to the exchange rate of Chinese RMB to U.S. dollar in 2008 ( $\left.1 \mathrm{RMB}=0.1611 \mathrm{USD}\right), 1000 \mathrm{RMB}=161.1 \mathrm{USD}$, $3000 \mathrm{RMB}=483.3 \mathrm{USD}$.

'Others included unmarried, separated, divorced and widowed. 

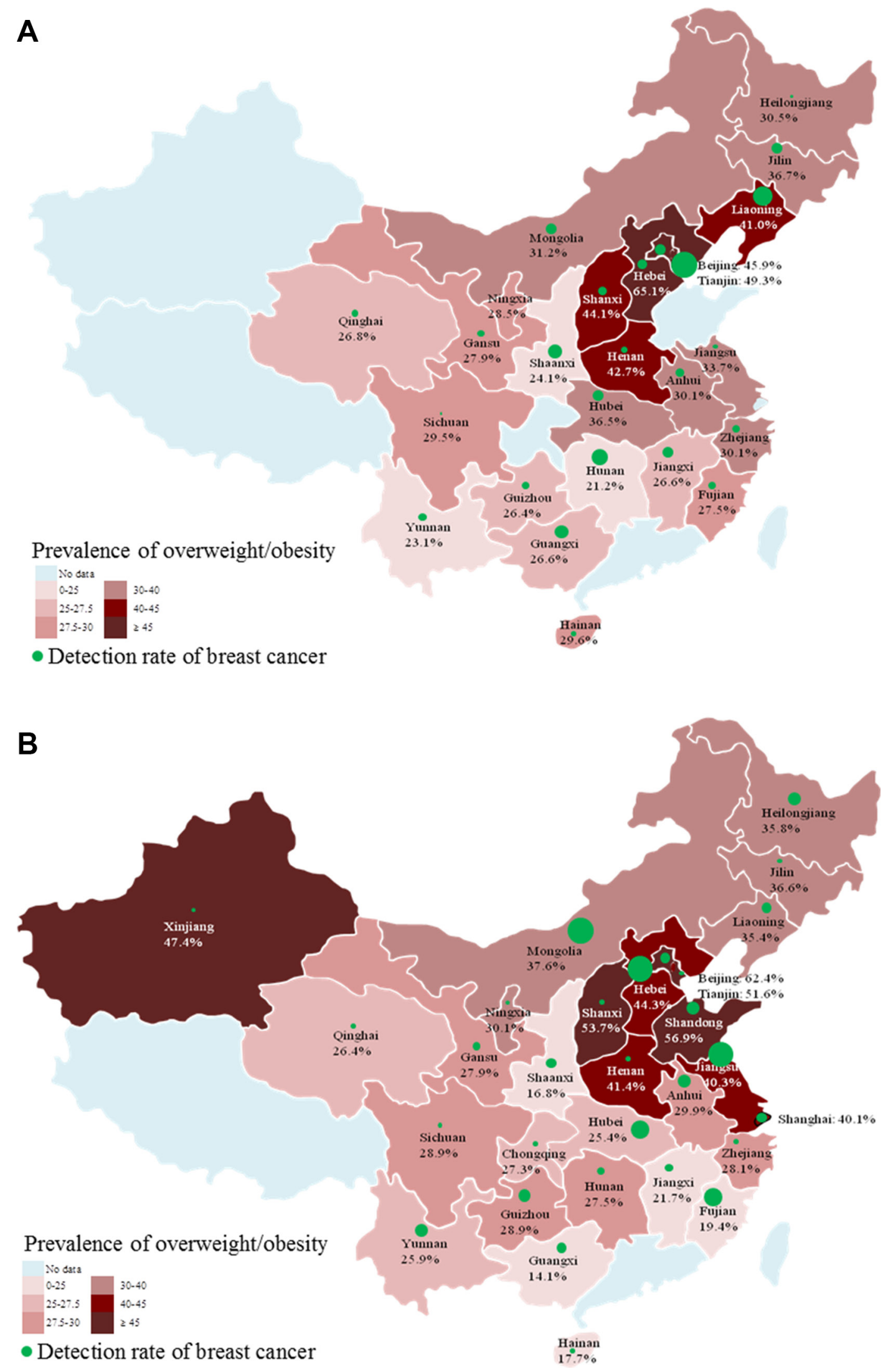

Figure 3: The provincial prevalence of overweight/obesity and the corresponding detection rate of breast cancer $\left(1 / 10^{5}\right)$ among Chinese urban (A) and rural (B) women. Note: The size of green bubbles corresponded with the size of provincial detection rate of breast cancer. 
Table 4: Trends between overweight/obesity and risk of breast cancer among Chinese urban and rural women

\begin{tabular}{|c|c|c|c|c|c|c|}
\hline \multirow{2}{*}{ BMI } & \multicolumn{3}{|c|}{ Breast cancer } & \multirow{2}{*}{$P_{1}$} & \multirow{2}{*}{$P_{2}$} & \multirow{2}{*}{ OR $(95 \% C I)$} \\
\hline & Case & Control & Detected rate $\left(1 / 10^{5}\right)$ & & & \\
\hline \multicolumn{7}{|l|}{ Urban } \\
\hline Normal $(\mathrm{BMI} \geq 18.5 \&<24.0)$ & 117 & 246182 & 47.5 & \multirow{3}{*}{0.004} & Ref. & 1.00 \\
\hline Underweight $(\mathrm{BMI}<18.5)$ & 8 & 18382 & 43.5 & & 0.81 & $0.92(0.45-1.88)$ \\
\hline Overweight (BMI $\geq 24.0 \&<28.0)$ & 75 & 110008 & 68.1 & & 0.02 & $1.44(1.10-1.92)$ \\
\hline Obesity (BMI $\geq 28.0$ ) & 22 & 22625 & 97.1 & \multirow{3}{*}{0.001} & 0.002 & $2.05(1.30-3.23)$ \\
\hline Less weight/Normal & 125 & 264564 & 47.2 & & Ref. & 1.00 \\
\hline Overweight/Obesity & 97 & 132633 & 73.1 & & 0.001 & $1.55(1.19-2.02)$ \\
\hline \multicolumn{7}{|l|}{ Rural } \\
\hline Normal $(\mathrm{BMI} \geq 18.5 \&<24.0)$ & 256 & 496314 & 51.6 & \multirow{4}{*}{0.77} & Ref. & 1.00 \\
\hline Underweight $(\mathrm{BMI}<18.5)$ & 17 & 30631 & 55.5 & & 0.77 & $1.08(0.66-1.76)$ \\
\hline Overweight (BMI $\geq 24.0 \&<28.0$ ) & 134 & 237026 & 56.5 & & 0.39 & $1.10(0.89-1.35)$ \\
\hline Obesity (BMI $\geq 28.0$ ) & 23 & 48942 & 47.0 & & 0.67 & $0.91(0.60-1.40)$ \\
\hline Less weight/Normal & 273 & 526945 & 51.8 & \multirow{2}{*}{0.56} & Ref. & 1.00 \\
\hline Overweight/Obesity & 157 & 285968 & 54.9 & & 0.56 & $1.06(0.87-1.29)$ \\
\hline
\end{tabular}

BMI, body mass index.

$P_{1}, P$ value of chi-square test.

$P_{2}, P$ value of univariate logistic regression.

the different social economic level between the urban areas and rural areas in China. As reported in the previous results, the urban women were more likely to have a high level of education, an occupation, high family income, few family residents and marriage at an older age when compared with rural women. These different urban-rural social economic levels could lead to different urbanrural lifestyle and dietary pattern. As pointed out in the previous studies, the dietary pattern shifted away from high-carbohydrate diets toward high-fat, high energydensity foods as income improved, particularly in the low- and middle-income group [28-30]. This phenomenon was worse in Chinese rural women. As reported from the Chinese National Nutritional Survey in 1982 and in 2002, the daily dietary fat intake increased $33 \mathrm{~g}$ for Chinese rural women (17g for urban women) during the 20 years, and the increments of daily dietary meat and animal oil were $46 \mathrm{~g}$ and $0.9 \mathrm{~g}$ ( $42 \mathrm{~g}$ and decrement of $0.2 \mathrm{~g}$ for urban women), respectively [31]. Moreover, the available evidences also suggested that higher income indicated more chance of accessing the healthier diet $[16,32]$. However, for Chinese rural population, although the increasing family income allows them to access more food, they spend more money on high-fat and high-energy foods rather than on a healthier diet [6]. That's why there was a negative association between high family income and overweight/obesity for urban women, but a positive association for rural women. In addition, this urban-rural disparity of overweight/obesity may also be a result of differences in knowledge and lifestyles between Chinese urban and rural women. Chinese rural women were more likely to live with their descendants and spend more time on taking care of their descendants when compared with their urban counterparts. Furthermore, in Chinese culture, there is a widespread belief that obesity represents health and prosperity [8]. This belief is more popular among rural women than urban women. Therefore, rural women paid less attention to their weight gain than their urban counterparts.

Another important finding was that potential urbanrural disparity of breast cancer could be the result of urban-rural disparity of overweight/obesity distribution. Although obesity was a known risk factor for breast cancer, the pattern of urban-rural disparity in overweight/ obesity distribution (high prevalence for rural women and low prevalence for urban women) was not very similar with the pattern of urban-rural disparity in breast cancer (positive trend between breast cancer and overweight/ obesity for urban women but a non-significant positive trend for rural women). These results implicated that future strategies focused on controlling overweight/ obesity could also prevent the breast cancer for Chinese urban women, but other strategies besides controlling overweight/obesity should also be emphasized to prevent breast cancer.

This study has several strengths. Firstly, the survey was population-based and the sample size was enough, which allowed a relatively stable estimation for each 
analysis and stratified analysis according to different demographic variables. Secondly, various training courses and seminars were conducted to ensure standardization of data collection. However, there were also several limitations in this study. Firstly, due to lack of information in dietary habits, sedentary behavior, and physical activity for urban and rural women, we cannot further explore the association between overweight/obesity risk and this information. Secondly, as described in the section of results, due to different sampling frame between this study and previously national representative surveys, results cannot be directly compare with previously national representative data in China. However, the data across these surveys could also remind us the crude increasing trend of overweight/obesity prevalence.

\section{MATERIALS AND METHODS}

\section{Study population}

The Chinese Ministry of Health launched the Chinese National Breast Cancer Screening Program (CNBCSP) which covered 29 provinces/autonomous regions/municipalities since 2008 . The program recruited 397 thousand urban women aged 35-69 years and 813 thousand rural women aged 35-59 years. The target population was clustered and selected from two or four communities (one or two communities for either urban areas or rural areas) with high incidence of breast cancer in each province. The targeted communities were chosen by the provincial cancer registry according to the local cancer registry data. The study was approved by Institutional Review Board of Tianjin Medical University Cancer Institute and Hospital, and all participants provided written informed consent.

\section{Exclusion criteria}

In this trial, asymptomatic women who had lived in their residential communities for $\geq 3$ years. The pregnant women and lactating less than 6 months women were excluded. Moreover, women who had previously been diagnosed with breast cancer were also excluded.

\section{Data collection}

During the face-to-face interview, demographic data was collected from all the participants with a structured questionnaire. The information included the participants' age, nationality, region, education, occupation, family income, number of family residents, health insurance, marriage status, and age at marriage.

Height was measured to the nearest $0.1 \mathrm{~cm}$ without shoes using a portable stadiometer and weight was measured to the nearest $0.1 \mathrm{~kg}$ with lightweight clothing on a calibrated beam scale. BMI was calculated as weight in kilograms divided by the square of height in meters $\left(\mathrm{Kg} / \mathrm{m}^{2}\right)$. Height and weight were measured by local trained investigators. In the final analyses, subjects who had a BMI either less than 14.0 or more than 50.0 were excluded [5].

\section{BMI categories}

According to the Chinese Working Group on Obesity, BMI $<18.5 \mathrm{~kg} / \mathrm{m}^{2}$ was considered underweight, BMI ranged from $18.5 \mathrm{~kg} / \mathrm{m}^{2}$ to $23.9 \mathrm{~kg} / \mathrm{m}^{2}$ was normal, $24.0-27.9 \mathrm{~kg} / \mathrm{m}^{2}$ was overweight and $28.0 \mathrm{~kg} / \mathrm{m}^{2}$ or greater was obesity [33]. In order to provide a comparable data with other regions around the world, the definitions according to WHO suggestions (underweight: BMI $<18.5 \mathrm{~kg} / \mathrm{m}^{2}$; normal: BMI $\geq 18.5 \mathrm{~kg} / \mathrm{m}^{2} \&<25.0 \mathrm{~kg} / \mathrm{m}^{2}$; overweight: $\mathrm{BMI} \geq 25.0 \mathrm{~kg} / \mathrm{m}^{2} \&<30.0 \mathrm{~kg} / \mathrm{m}^{2}$; obesity: $\mathrm{BMI} \geq 30.0 \mathrm{~kg} / \mathrm{m}^{2}$ ) were also used [34].

\section{Quality control}

Various training courses and seminars according to the study protocol were conducted with a train-thetrainer model [35]. Firstly, all provincial investigators who participated in the study were trained to learn the standardized epidemiological interview. Secondly, the local investigators who participated in the study were trained by the provincial investigators. Regular intensive training was also provided for local investigators to ensure adherence to protocols.

All the examination data was double-entered and checked for its consistency. Further comprehensive logic checking was conducted to remove the logic errors between variables before analysis.

\section{Statistical analysis}

Chi-square tests were used to compare the overweight/obesity distribution between different demographic characteristics for urban and rural women. Chi-squared test for trend was used to test whether there was a linear trend between overweight/obesity prevalence and ordinal categorical variable. Odds ratios and 95\% CI calculated with univariate logistic regression were used to evaluate the association between demographic characteristics and risk of overweight/obesity, and the trend between overweight/obesity and the risk of breast cancer. All analyses were conducted with SPSS version 19.0. $P$ value $<0.05$ was considered to be statistically significant.

\section{CONCLUSIONS}

In summary, this study demonstrated an obvious urban-rural disparity in the prevalence of overweight/ obesity. And this urban-rural disparity in overweight/ 
obesity distribution could also lead to a potential disparity in breast cancer distribution. More worrisomely, compared with previously national representative data in China, the prevalence of overweight/obesity could increase in the future China. This trend could lead to an increasing burden of obesity and obesity associated diseases, such as breast cancer. Therefore, in the future, different strategies targeting different influential factors of overweight/ obesity are needed for Chinese urban and rural women so as to control the increasing burden of obesity and obesity associated diseases.

\section{CONFLICTS OF INTEREST}

The authors declare no conflicts of interest.

\section{GRANT SUPPORT}

This work was supported by the Chinese National Key Scientific and Technological Project (Grants 2014BAI09B09 and 2015BAI12B15), National Natural Science Foundation of China (Grants 81473039, 81502476, and 71273187), the Program for Changjiang Scholars and Innovative Research Team in University in China (IRT_14R40 to K.C.).

\section{REFERENCES}

1. Renehan AG, Tyson M, Egger M, Heller RF, Zwahlen M. Body-mass index and incidence of cancer: a systematic review and meta-analysis of prospective observational studies. Lancet. 2008; 371:569-578.

2. Ng M, Fleming T, Robinson M, Thomson B, Graetz N, Margono C, Mullany EC, Biryukov S, Abbafati C, Abera SF, Abraham JP, Abu-Rmeileh NM, Achoki T, et al. Global, regional, and national prevalence of overweight and obesity in children and adults during 1980-2013: a systematic analysis for the Global Burden of Disease Study 2013. Lancet. 2014; 384:766-781.

3. Forouzanfar MH, Alexander L, Anderson HR, Bachman VF, Biryukov S, Brauer M, Burnett R, Casey D, Coates MM, Cohen A, Delwiche K, Estep K, Frostad JJ, et al. Global, regional, and national comparative risk assessment of 79 behavioural, environmental and occupational, and metabolic risks or clusters of risks in 188 countries, 1990-2013: a systematic analysis for the Global Burden of Disease Study 2013. Lancet. 2015; 386:2287-2323.

4. Arnold M, Pandeya N, Byrnes G, Renehan AG, Stevens GA, Ezzati M, Ferlay J, Miranda JJ, Romieu I, Dikshit R, Forman D, Soerjomataram I. Global burden of cancer attributable to high body-mass index in 2012: a population-based study. Lancet Oncol. 2015; 16:36-46.

5. Zheng W, McLerran DF, Rolland B, Zhang X, Inoue M, Matsuo K, He J, Gupta PC, Ramadas K, Tsugane S, Irie F, Tamakoshi A, Gao YT, et al. Association between body-mass index and risk of death in more than 1 million Asians. N Engl J Med. 2011; 364:719-729.

6. Wang Y, Mi J, Shan XY, Wang QJ, Ge KY. Is China facing an obesity epidemic and the consequences? The trends in obesity and chronic disease in China. Int J Obes (Lond). 2007; 31:177-188.

7. Wang JH, Zhai F, Du SF, Wang ZH, He YN. The trends of prevalence of obesity in adults in part areas of China. Acta Nutrimenta Sinica. 2004; 26:329-332.

8. Wu Y. Overweight and obesity in China. BMJ. 2006; 333:362-363.

9. Xi B, Liang YJ, He TP, Reilly KH, Hu Y, Wang Q, Yan Y, Mi J. Secular trends in the prevalence of general and abdominal obesity among Chinese adults, 1993-2009. Obes Res. 2012; 13:287-296.

10. Gu D, Reynolds K, Wu X, Chen J, Duan X, Reynolds RF, Whelton PK, He J. Prevalence of the metabolic syndrome and overweight among adults in China. Lancet. 2005; 365:1398-1405.

11. Weinsier RL, Johnston MH, Doleys DM, Bacon JA. Dietary management of obesity: evaluation of the time-energy displacement diet in terms of its efficacy and nutritional adequacy for long-term weight control. Br J Nutr. 1982; 47:367-379.

12. Pi-Sunyer FX. Dietary practices in obesity. Bull N Y Acad Med. 1982; 58:263-274.

13. Sobal J, Stunkard AJ. Socioeconomic status and obesity: a review of the literature. Psychol Bull. 1989; 105:260-275.

14. Borders TF, Rohrer JE, Cardarelli KM. Gender-specific disparities in obesity. J Community Health. 2006; 31:57-68.

15. Sabanayagam C, Shankar A, Wong TY, Saw SM, Foster PJ. Socioeconomic status and overweight/obesity in an adult Chinese population in Singapore. J Epidemiol. 2007; 17:161-168.

16. McLaren L. Socioeconomic status and obesity. Epidemiol Rev. 2007; 29:29-48.

17. Jackson JE, Doescher MP, Jerant AF, Hart LG. A national study of obesity prevalence and trends by type of rural county. J Rural Health. 2005; 21:140-148.

18. Patterson PD, Moore CG, Probst JC, Shinogle JA. Obesity and physical inactivity in rural America. J Rural Health. 2004; 20:151-159.

19. Befort CA, Nazir N, Perri MG. Prevalence of obesity among adults from rural and urban areas of the United States: findings from NHANES (2005-2008). J Rural Health. 2012; 28:392-397.

20. Kettle SM, Roebothan BV, West R. Prevalence of specific cardiovascular disease risk factors in young Newfoundland and Labrador adults living in urban and rural communities. Can J Rural Med. 2005; 10:81-85.

21. Ebrahim S, Kinra S, Bowen L, Andersen E, Ben-Shlomo Y, Lyngdoh T, Ramakrishnan L, Ahuja RC, Joshi P, Das SM, Mohan M, Davey SG, Prabhakaran D. The effect of ruralto-urban migration on obesity and diabetes in India: a crosssectional study. PLoS Med. 2010; 7:e1000268. 
22. Seubsman SA, Lim LL, Banwell C, Sripaiboonkit N, Kelly M, Bain C, Sleigh AC. Socioeconomic status, sex, and obesity in a large national cohort of 15-87-year-old open university students in Thailand. J Epidemiol. 2010; 20:13-20.

23. Dahly DL, Gordon-Larsen P, Popkin BM, Kaufman JS, Adair LS. Associations between multiple indicators of socioeconomic status and obesity in young adult Filipinos vary by gender, urbanicity, and indicator used. J Nutr. 2010; 140:366-370.

24. Zhai F, Wang H, Du S, He Y, Wang Z, Ge K, Popkin BM. Prospective study on nutrition transition in China. Nutr Rev. 2009; 67:S56-61.

25. Chen CM, Zhao W, Yang Z, Zhai Y, Wu Y, Kong L. The role of dietary factors in chronic disease control in China. Obes Rev. 2008; 9:100-103.

26. Neuman M, Kawachi I, Gortmaker S, Subramanian SV. Urban-rural differences in BMI in low- and middle-income countries: the role of socioeconomic status. Am J Clin Nutr. 2013; 97:428-436.

27. WHO/FAO Expert Consultation on Diet, Nutrition and the Prevention of Chronic Disease. Diet, Nutrition and the Prevention of Chronic Disease. Report of the Joint WHO/FAO Expert Consultation. Geneva: World Health Organization, 2003.

28. Carlo WA, Goudar SS, Jehan I, Chomba E, Tshefu A, Garces A, Parida S, Althabe F, McClure EM, Derman RJ,
Goldenberg RL, Bose C, Krebs NF, et al. Newborn-care training and perinatal mortality in developing countries. $\mathrm{N}$ Engl J Med. 2010; 362:614-623.

29. Krueger PM, Coleman MK, Rooks RN. Race/ethnicity, nativity and trends in BMI among U.S. adults. Obesity (Silver Spring). 2014; 22:1739-1746.

30. Popkin BM, Horton S, Kim S, Mahal A, Shuigao J. Trends in diet, nutritional status, and diet-related noncommunicable diseases in China and India: the economic costs of the nutrition transition. Nutr Rev. 2001; 59:379-390.

31. Du S, Mroz TA, Zhai F, Popkin BM. Rapid income growth adversely affects diet quality in China - particularly for the poor!. Soc Sci Med. 2004; 59:1505-1515.

32. Du S, Lu B, Zhai F, Popkin BM. A new stage of the nutrition transition in China. Public Health Nutr. 2002; 5:169-174.

33. Zhou BF. Predictive values of body mass index and waist circumference for risk factors of certain related diseases in Chinese adults - study on optimal cut-off points of body mass index and waist circumference in Chinese adults. Biomed Environ Sci. 2002; 15:83-96.

34. Zhai FY, He YN, Ma GS, Li YP, Wang ZH, Hu YS, Zhao LY, Cui ZH, Li Y, Yang XG. Study on the current status and trend of food consumption among Chinese population. Chin J Epidemiol. 2005; 26:485-488.

35. Power EM. Determinants of healthy eating among lowincome Canadians. Can J Public Health. 2005; 96:S37-42, S42-8. 